\title{
Nucleon structure with partially twisted boundary conditions
}

QCDSF and UKQCD Collaborations: M. Göckeler, ${ }^{a}$ Ph. Hägler ${ }^{*}{ }^{b}$ R. Horsley, ${ }^{c}$ Y. Nakamura, ${ }^{d}$ M. Ohtani, ${ }^{e}$ D. Pleiter, ${ }^{d}$ P.E.L. Rakow,${ }^{f}$ A. Schäfer, ${ }^{a}$ G. Schierholz,,${ }^{a} d$ H. Stüben ${ }^{g}$ and J.M. Zanotti ${ }^{c}$

${ }^{a}$ Institut für Theoretische Physik, Universität Regensburg, 93040 Regensburg, Germany

${ }^{b}$ Institut für Theoretische Physik T39, Physik-Department der TU München, James-Franck-Straße, 85747 Garching, Germany

${ }^{c}$ School of Physics and Astronomy, University of Edinburgh, Edinburgh EH9 3JZ, UK

${ }^{d}$ John von Neumann-Institut für Computing NIC / DESY, 15738 Zeuthen, Germany

${ }^{e}$ Kyorin University, Department of Physics, 6-20-2 Shinkawa Mitaka-shi, Tokyo 181, Japan

${ }^{f}$ Theoretical Physics Division, Department of Mathematical Sciences, University of Liverpool, Liverpool L69 3BX, UK

${ }^{g}$ Konrad-Zuse-Zentrum für Informationstechnik Berlin, 14195 Berlin, Germany

Email: phaeglereph.tum.de

\begin{abstract}
A large number of fundamental hadron structure observables are defined in the limit of vanishing momentum transfer, but at the same time cannot be directly extracted from forward matrix elements. This is a challenge for current lattice QCD simulations, where volumes and lattice spacings are such that the lowest accessible non-zero momentum transfers are $\sim 0.15 \mathrm{GeV}^{2}$ and larger, making in general model-dependent extrapolations to the forward limit necessary. Twisted boundary conditions for the valence quarks provide the opportunity to study hadronic matrix elements in dynamical lattice QCD calculations for almost arbitrary hadron momenta. We present preliminary results for the Dirac- and Pauli form factors and the form factors of the energy momentum tensor for the nucleon very close to the forward limit, using partially twisted boundary conditions. The calculations are based on gauge configurations generated with two flavors of clover-improved Wilson fermions.
\end{abstract}

The XXVI International Symposium on Lattice Field Theory

July 14-19 2008

Williamsburg, Virginia, USA

\footnotetext{
* Speaker.
} 


\section{Introduction}

Form factors (FFs) and generalized form factors (GFFs), i.e. moments of generalized parton distributions, play a central role in our attempt to unravel the inner structure of hadrons ${ }^{1}$. They give direct access to a number of fundamental observables, for example charge radii, $\left\langle r_{i}^{2}\right\rangle \propto$ $\left.\frac{d}{d Q^{2}} F_{i}\left(Q^{2}\right)\right|_{Q^{2}=0}$, anomalous magnetic moments, $\kappa=F_{2}\left(Q^{2}=0\right)$, quadrupole moments of hadrons with spin $>1 / 2$, and angular momentum contributions to the nucleon spin [2], $J=\left(A_{20}(t=\right.$ $\left.0)+B_{20}(t=0)\right) / 2$, which are all defined at zero momentum transfer, but require for their numerical analysis small non-zero values of $t=q^{2}=-Q^{2}$. We note in particular that the nucleon (G)FFs $F_{2}\left(Q^{2}\right), B_{20}(t)$ and $C_{20}(t)$ appear in the parametrization of matrix elements of local quark operators always together with prefactors of $\Delta=q=P^{\prime}-P$ and therefore cannot be extracted in the forward limit, $P^{\prime}=P$. For example we have

$$
\left\langle N\left(P^{\prime}\right)\left|T_{q}^{\mu \nu}\right| N(P)\right\rangle=\bar{U}\left(P^{\prime}\right)\left\{\gamma^{\{\mu} \bar{P}^{v\}} A_{20}^{q}(t)+\frac{i \bar{P}^{\{\mu} \sigma^{\nu\} \rho} \Delta_{\rho}}{2 m_{N}} B_{20}^{q}(t)+\frac{\Delta^{\mu} \Delta^{v}}{m_{N}} C_{20}^{q}(t)\right\} U(P),(1
$$

where $T_{q}^{\mu v}$ is the QCD energy momentum tensor of quarks and $\{\cdots\}$ denotes symmetrization. Momenta in lattice simulations are discrete, $\vec{p}=(2 \pi /(a L)) \vec{n}$, and typical lowest non-zero momentum components for spatial lattice extents $L$ and lattice spacings $a$ of current simulations are $p=$ $2 \pi /(a L) \sim 0.3-0.5 \mathrm{GeV}$, leading to rather large minimal momentum transfers of $|t|_{\min }^{\neq 0} \sim 0.15-$ $0.4 \mathrm{GeV}^{2}$. Hence lattice studies of many important observables require extrapolations to $t=0$, which depend in general on the chosen ansatz for the $t$-dependence, e.g. a monopole or dipole form, introducing additional systematic uncertainties. Furthermore, chiral extrapolations of lattice results based on chiral perturbation theory (ChPT) predictions for the simultaneous dependence on $m_{\pi}$ and $t$ (see, e.g., [3,4]) can only be safely performed for small values of the pion mass and the momentum transfer. Finally, due to the discrete lattice momenta, neighboring values of $t$ are often separated by large gaps of up to $\sim 0.5 \mathrm{GeV}^{2}$, amplifying the dependence on the parametrizations of the form factors to interpolate between the lattice data points. All this provides strong motivation for the use of partially twisted boundary conditions (pTBCs), introduced by Sachrajda and Villadoro [5] and Bedaque and Chen [6], in unquenched lattice QCD studies. Twisted spatial boundary conditions for the valence quarks, $q_{f, i}\left(\vec{x}+L \vec{e}_{j}\right)=\exp \left(i\left(\vec{\theta}_{f, i}\right)_{j}\right) q_{f, i}(\vec{x})$ with $\left(\vec{\theta}_{f, i}\right)_{j}=0, \ldots, 2 \pi, j=1,2,3$, as illustrated in Fig. 1, provide in principle access to arbitrary values of the ini-

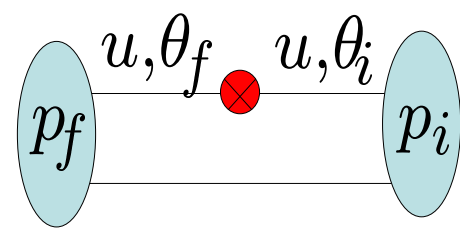

Figure 1: Illustration of pTBCs. tial, $\vec{p}_{i}$, and final, $\vec{p}_{f}$, momenta and thereby the momentum transfer by tuning of the twisting angles $\vec{\theta}_{f, i}$. For, e.g., zero Fourier momenta, $\vec{p}_{f, i}^{F}=(2 \pi /(a L)) \vec{n}_{f, i}=0$, one has $t^{\mathrm{TBC}}=$ $\left(E^{\mathrm{TBC}}\left(\overrightarrow{\boldsymbol{\theta}}_{f}\right)-E^{\mathrm{TBC}}\left(\overrightarrow{\boldsymbol{\theta}}_{i}\right)\right)^{2}-\left(\overrightarrow{\boldsymbol{\theta}}_{f}-\overrightarrow{\boldsymbol{\theta}}_{i}\right)^{2} /(a L)^{2}$, where $E^{\mathrm{TBC}}(\overrightarrow{\boldsymbol{\theta}})^{2}=m^{2}+\overrightarrow{\boldsymbol{\theta}}^{2} /(a L)^{2}$. It was shown in $[5,6]$ that finite volume effects in partially twisted QCD are in general exponentially suppressed, i.e. $\propto \exp \left(-m_{\pi} L\right)$. Partially TBCs were studied for the pion in the framework of partially quenched ChPT in [7] for general kinematics where isospin symmetry breaking effects are present, which can however be avoided by working in the Breit frame, $\vec{\theta}_{f}=-\vec{\theta}_{i}$, as shown in [8]. In either case, finite

\footnotetext{
${ }^{1}$ For an overview of recent progress in hadron structure calculations in lattice QCD, see [1].
} 


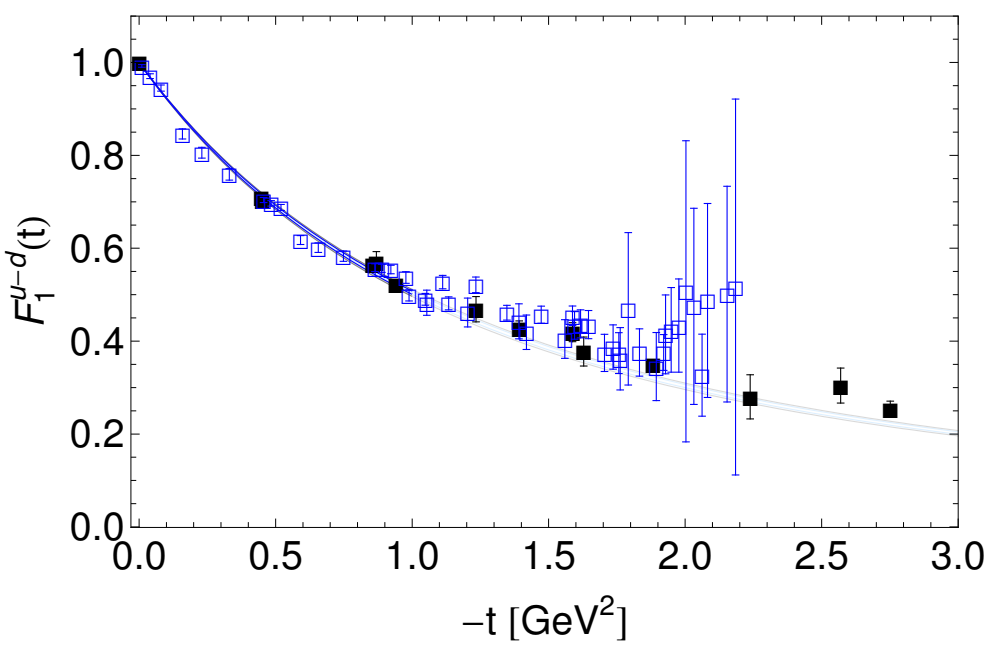

Figure 2: Dirac form factor $F_{1}$ of the nucleon in the isovector channel for $m_{\pi} \sim 630 \mathrm{MeV}$. The gray error band represents a fit to the filled data points obtained for periodic boundary conditions. A fit to all data points with $|t| \leq 1 \mathrm{GeV}^{2}$ is represented by the blue error band.

volume effects are predicted to be small for the pion form factor in current lattice QCD simulations with pTBCs. Finite volume corrections from pTBCs for the nucleon isovector magnetic moment $\kappa^{u-d}=F_{2}^{u-d}(0)$ have been studied in partially quenched ChPT in [9], where effects as large as $\sim 20 \%$ were found for small twisting angles, at pion masses of $\sim 350 \mathrm{MeV}$ and in volumes of $\sim(2.5 \mathrm{fm})^{2}$. Since these ChPT results are only valid at low pion masses, we unfortunately cannot directly use them to identify and correct for possible finite volume errors in our preliminary computations of $F_{2}$ at a pion mass of $\sim 630 \mathrm{MeV}$ that will be presented below, but we have to keep in mind that these effects may represent a significant source of uncertainty. On the upside, within its region of applicability, ChPT allows for a model-independent, quantitative study of the finite size effects without introducing any additional low energy constants and may therefore be of great help in the analysis of nucleon isovector FFs in future lattice calculations. We note that pTBCs have already been successfully employed in lattice studies of the $K \rightarrow \pi$ semileptonic form factor and the pion form factor [10-12].

We have performed lattice calculations of nucleon FFs and GFFs with pTBCs in the framework of simulations with $n_{f}=2$ flavors of non-perturbatively clover-improved Wilson fermions and Wilson glue. The QCDSF/UKQCD collaborations have generated configurations for four different couplings $\beta=5.20,5.25,5.29,5.40$ with up to six different $\kappa=\kappa_{\text {sea }}$ values per $\beta$, corresponding to lattice spacings as small as $\sim 0.07 \mathrm{fm}$ and pion masses as low as $\sim 260 \mathrm{MeV}$. The nucleon mass was employed to set the scale, and a chirally extrapolated $r_{0} / a$ was used to transform results from lattice to physical units. In this contribution, we will show preliminary results obtained for an ensemble with $\beta=5.29$ and $\kappa=0.13590$, corresponding to a lattice spacing of $\sim 0.075 \mathrm{fm}$, a pion mass of $m_{\pi} \approx 630 \mathrm{MeV}$, and a spatial volume $V=L^{3}$ with $m_{\pi} L \approx 5.7$. The lattice operators that are required for the analysis of the GFFs $A_{20}, B_{20}$ and $C_{20}$ were non-perturbatively renormalized [13], and the results have been transformed to the $\overline{\mathrm{MS}}$ scheme at a scale of $\mu=2 \mathrm{GeV}$. Some details about the simulations and the extraction of the nucleon FFs and GFFs in lattice QCD can be found in, e.g., [14-17]. We have implemented the TBCs for the valence quark fields by using modified 


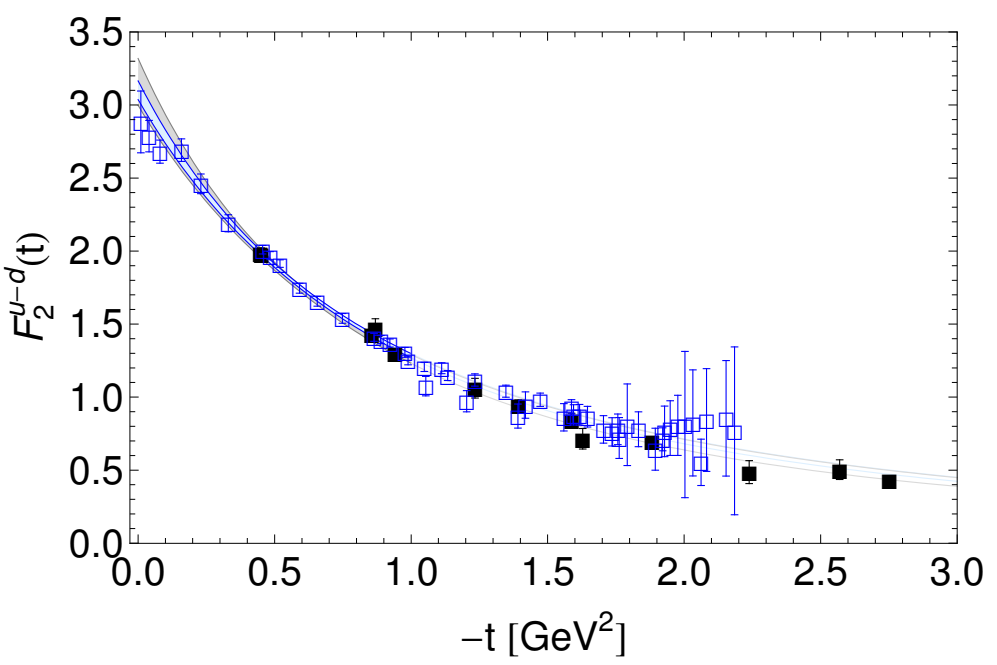

Figure 3: Pauli form factor $F_{2}$ of the nucleon in the isovector channel for $m_{\pi} \sim 630 \mathrm{MeV}$. For an explanation of the error bands see caption of Fig. 2.

link variables in the calculation of the quark propagators, as described in [18].

\section{Simulation results for the Dirac and Pauli form factors}

Figure 2 displays results for the Dirac form factors of the nucleon in the isovector channel. The smaller number of filled squares represent results obtained for standard periodic boundary conditions in the sea and valence quark sectors. Lattice data points obtained for pTBCs are given by the open squares. The twisting angles were tuned such that a very small non-zero $t=-Q^{2}$ could be accessed, in this case $|t|_{\min }^{\neq 0} \approx 0.01 \mathrm{GeV}^{2}$, and that the gaps between the $t$-values corresponding to the Fourier-momenta $\vec{p}^{F}=(2 \pi /(a L)) \vec{n}$ could be approximately evenly filled. A linear fit, $F(t)=$ $F(0)+\left\langle r_{1}^{2}\right\rangle t / 6$, to the four lattice data points with $|t| \leq 0.1 \mathrm{GeV}^{2}$ gives a mean square radius of $\left\langle r_{1}^{2}\right\rangle=0.164(24) \mathrm{fm}^{2}$, a value that is rather small and shows a comparatively large error $>10 \%$. In an alternative approach, the filled data points, and the combined filled and open data points were fitted separately using a dipole ansatz,

$$
F(t)=\frac{F(0)}{\left(1-t / m_{D}^{2}\right)^{2}}
$$

with free parameters $F(0)$ and $m_{D}$, as represented by the solid lines and shaded bands in Fig. 2. From such fits to the lattice data in the range $|t|=0, \ldots, 1 \mathrm{GeV}^{2}$, we find a mean square radius of $\left\langle r_{1}^{2}\right\rangle^{u-d}=12 / m_{D}^{2}=0.191(2) \mathrm{fm}^{2}$ for the combined lattice data points, compared to $\left\langle r_{1}^{2}\right\rangle^{u-d}=$ $0.191(5) \mathrm{fm}^{2}$ for the Fourier momentum (periodic BCs) based results. This indicates that pTBCs could help to substantially reduce the error in the lattice determination of such a fundamental observable. We note, however, that individual groups of pTBC data points, e.g. in the region $t=-0.15, \ldots,-0.35 \mathrm{GeV}^{2}$, seem to be systematically lower than the average trend represented by the error bands. This may indicate the presence of discretization errors and/or finite volume effects due to the pTBCs. Corresponding results for the Pauli form factor are shown in Fig. 3. At 


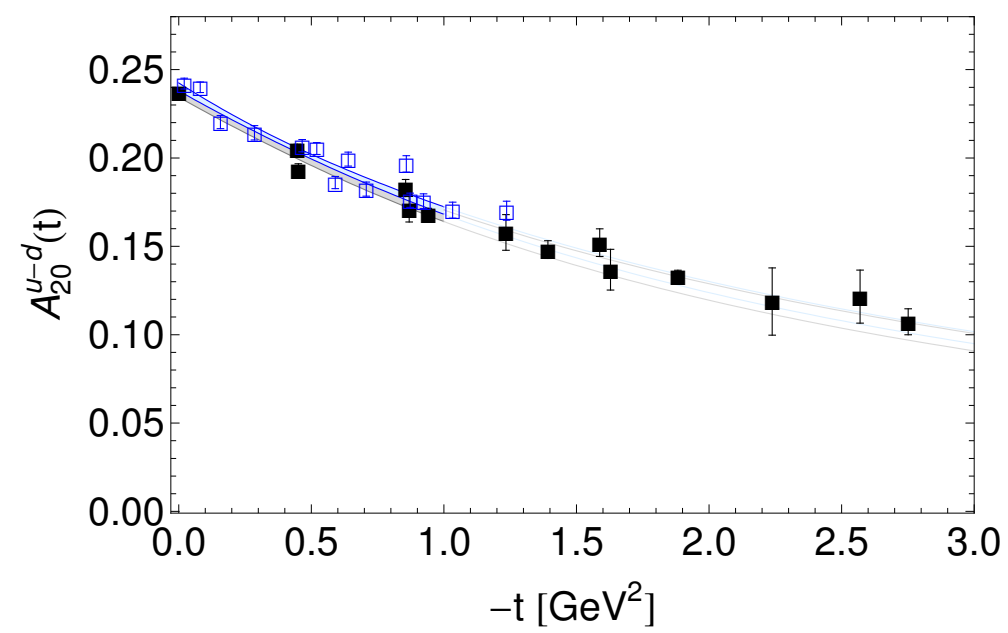

Figure 4: Generalized form factor $A_{20}$ of the nucleon in the isovector channel for $m_{\pi} \sim 630 \mathrm{MeV}$. For an explanation of the error bands see caption of Fig. 2.

the lowest accessible non-zero $t$, we find a value of $\kappa^{u-d} \approx F_{2}^{u-d}\left(t \approx-0.01 \mathrm{GeV}^{2}\right)=2.88(21)$. Although it is quite remarkable that a value with a statistical error below $10 \%$ could be obtained for a nearly vanishing momentum transfer using pTBCs, it should be noted that the three data points at $-t \approx 0.01,0.04$ and $0.08 \mathrm{GeV}^{2}$ seem to lie systematically below the average trend, as indicated by the error bands obtained from dipole fits to the lattice data for $|t| \leq 1 \mathrm{GeV}^{2}$. As before, this may be an indication for systematic uncertainties related to, e.g., finite size effects, which are not yet under control. From the dipole fits, we obtain $\left\langle r_{2}^{2}\right\rangle^{u-d}=0.259(10) \mathrm{fm}^{2}$ and an anomalous magnetic moment of $\kappa^{u-d}=3.101(64)$ for the combined lattice data, compared to $\left\langle r_{2}^{2}\right\rangle^{u-d}=0.272(26) \mathrm{fm}^{2}$ and $\kappa^{u-d}=3.158(160)$ from the fit excluding the pTBC results (represented by the open squares). Clearly, pTBCs offer a more accurate and statistically precise determination of, e.g., the anomalous magnetic moment $\kappa^{u-d}$, which cannot be extracted directly at $t=0$, however possible systematic uncertainties must be studied in some more detail before solid conclusions can be reached.

\section{Simulation results for the form factors of the energy momentum tensor}

We now turn to a brief discussion of the form factors of the energy momentum tensor, Eq. (1.1), in the isovector channel. The $t$-dependences of the GFFs $A_{20}^{u-d}, B_{20}^{u-d}$ and $C_{20}^{u-d}$ are shown in Figs. 4 , 5 and 6 , where the filled squares represent results obtained for periodic BCs, while the open squares correspond to values of $t$ that could be reached using pTBCs. Dipole fits, Eq. (2.1), restricted to $|t| \leq 1 \mathrm{GeV}^{2}$, were performed to the combined (filled and open squares) and the Fourier momentum results separately, and are represented by the lines and error bands. Results for $A_{20}^{u-d}$ obtained with pTBCs in Fig. 4 show some scatter but are overall compatible with the results from periodic BCs. Of particular interest with respect to the nucleon spin structure is the direct determination of $B_{20}^{u-d}(t)$ in Fig. 5 at very small momentum transfer, $t \approx-0.02 \mathrm{GeV}^{2}$, giving a value of $B_{20}^{u-d}\left(-0.02 \mathrm{GeV}^{2}\right)=0.402(39)$. It is very promising that a precision of $\sim 10 \%$ could be achieved so close to the forward limit. The dipole fits give somewhat larger values of $B_{20}^{u-d}(0)=0.440(19)$ and $B_{20}^{u-d}(0)=0.432(35)$, for the cases of the combined and the Fourier momentum lattice data, 


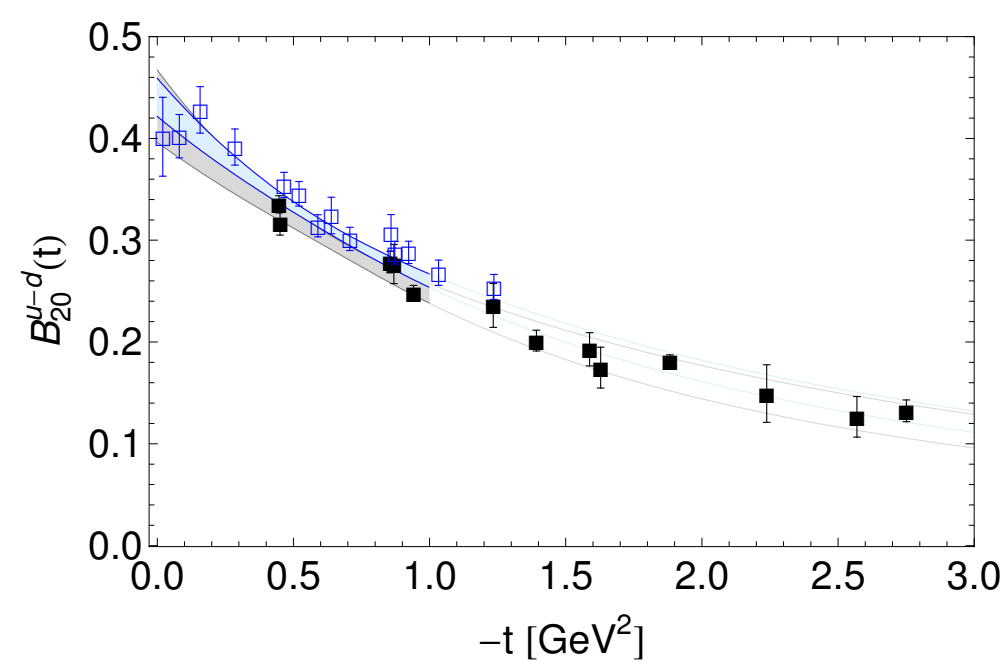

Figure 5: Generalized form factor $B_{20}$ of the nucleon in the isovector channel for $m_{\pi} \sim 630 \mathrm{MeV}$. For an explanation of the error bands see caption of Fig. 2.

respectively. Figure 6 shows that the inclusion of the pTBCs data points in the fit does not necessarily improve the statistical precision, but still may lead to a more accurate description of the $t$-dependence. While the filled squares are mostly compatible with zero in this case, the pTBCs results point towards small negative values of $C_{20}$ for $|t| \leq 1 \mathrm{GeV}^{2}$. Accordingly, the dipole fit to the full set of data points tends to negative values in the forward limit, though with rather large statistical uncertainty.

\section{Conclusions}

Employing partially twisted boundary conditions, we have performed a first direct calculation of the nucleon form factors $F_{1}$ and $F_{2}$ and of the form factors of the energy momentum tensor $A_{20}, B_{20}$ and $C_{20}$ in the isovector channel at very small non-zero values of the momentum transfer squared, $|t| \gtrsim 0.01 \mathrm{GeV}^{2}$. For the given ensemble with $m_{\pi} \approx 630 \mathrm{MeV}$ and $m_{\pi} L \approx 5.7$, we were able to achieve a good statistical precision of $\lesssim 10 \%$ for $F_{2}^{u-d}(t)$ and $B_{20}^{u-d}(t)$ very close to the forward limit at $-t \approx 0.01-0.02 \mathrm{GeV}^{2}$. Lattice data points obtained for pTBCs show in some cases more scatter and possible systematic deviations from the average trends, which may be an indication for discretization and finite volume effects [9]. These will have to be investigated further before firm conclusions can be drawn from our results concerning the structure of the nucleon at very low momentum transfer.

\section{Acknowledgments}

The numerical calculations have been performed on the Hitachi SR8000 at LRZ (Munich), the APE1000 and apeNEXT at NIC/DESY (Zeuthen) and the BlueGenes at NIC/Jülich and Edinburgh. This work was supported by the DFG (Forschergruppe Gitter-Hadronen-Phänomenologie, Transregional Collaborative Research Center SFB/TRR 55 and Emmy-Noether program) and by the EU I3HP under contract number RII3-CT-2004-506078. Ph.H. would like to thank the Excellence Cluster Universe at the TU Munich for support. 


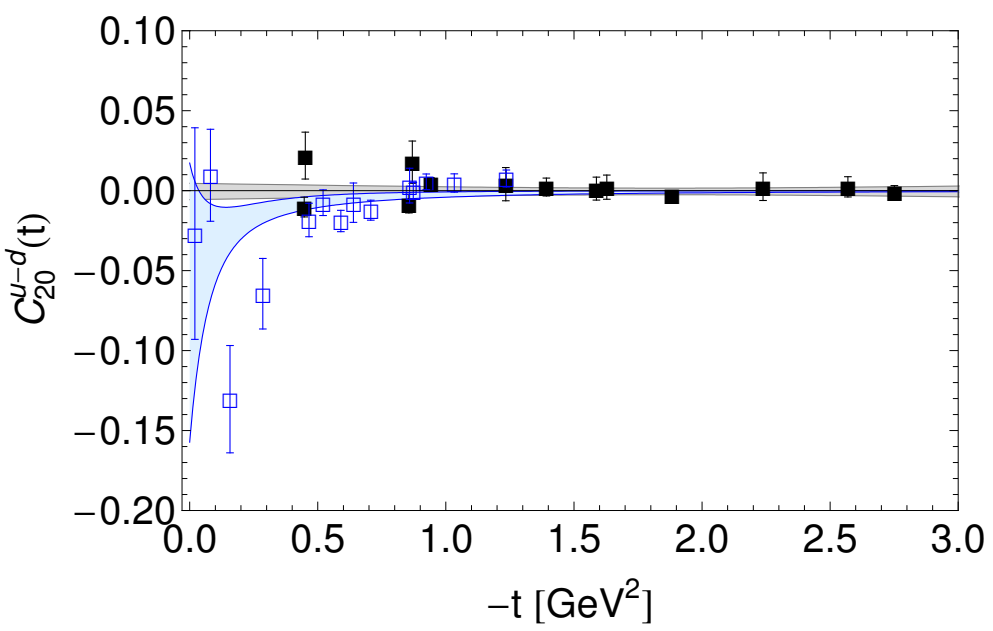

Figure 6: Generalized form factor $C_{20}$ of the nucleon in the isovector channel for $m_{\pi} \sim 630 \mathrm{MeV}$. For an explanation of the error bands see caption of Fig. 2.

\section{References}

[1] J. Zanotti, PoS LAT2008 (2008) 007.

[2] X. D. Ji, Phys. Rev. Lett. 78 (1997) 610 [arXiv:hep-ph/9603249].

[3] M. Göckeler et al. [QCDSF], Phys. Rev. D 71 (2005) 034508 [arXiv:hep-lat/0303019].

[4] M. Dorati, T. A. Gail and T. R. Hemmert, Nucl. Phys. A 798 (2008) 96 [arXiv:nucl-th/0703073].

[5] C. T. Sachrajda and G. Villadoro, Phys. Lett. B 609 (2005) 73 [arXiv:hep-lat/0411033].

[6] P. F. Bedaque and J. W. Chen, Phys. Lett. B 616 (2005) 208 [arXiv:hep-lat/0412023].

[7] F. J. Jiang and B. C. Tiburzi, Phys. Lett. B 645 (2007) 314 [arXiv:hep-lat/0610103].

[8] F. J. Jiang and B. C. Tiburzi, Phys. Rev. D 78 (2008) 037501 [arXiv:0806.4371 [hep-lat]].

[9] B. C. Tiburzi, Phys. Lett. B 641 (2006) 342 [arXiv:hep-lat/0607019].

[10] P. A. Boyle et al., JHEP 0705 (2007) 016 [arXiv:hep-lat/0703005].

[11] S. Simula [ETMC], PoS LAT2007 (2007) 371 [arXiv:0710.0097 [hep-lat]].

[12] P. A. Boyle et al., JHEP 0807 (2008) 112 [arXiv:0804.3971 [hep-lat]].

[13] G. Martinelli et al., Nucl. Phys. B 445 (1995) 81 [hep-lat/9411010]; M. Göckeler et al., Nucl. Phys. B 544 (1999) 699 [hep-lat/9807044].

[14] M. Göckeler et al., Nucl. Phys. Proc. Suppl. 153 (2006) 146 [arXiv:hep-lat/0512011].

[15] M. Göckeler et al., Eur. Phys. J. A 32 (2007) 445 [arXiv:hep-lat/0609001].

[16] D. Brömmel et al. [QCDSF-UKQCD], PoS LAT2007 (2007) 158 [arXiv:0710.1534 [hep-lat]].

[17] M. Göckeler et al. [QCDSF-UKQCD], PoS LAT2007 (2007) 161 [arXiv:0710.2159 [hep-lat]].

[18] J. M. Flynn, A. Jüttner and C. T. Sachrajda [UKQCD], Phys. Lett. B 632 (2006) 313 [arXiv:hep-lat/0506016]. 